DESY 10-172

SFB/CPP-10-90

$\mathrm{WUB} / 10-24$

BUW-SC 2010/5

\title{
Determination of the Static Potential with Dynamical Fermions
}

\author{
$\bar{\nexists} \perp_{\text {coliaboration }}$ \\ Michael Donnellan ${ }^{a}$, Francesco Knechtli ${ }^{b}$, Björn Leder ${ }^{b, c}$, Rainer Sommer ${ }^{a}$ \\ ${ }^{a}$ NIC, DESY, Platanenallee 6, D-15738 Zeuthen, Germany \\ ${ }^{b}$ Department of Physics, Bergische Universität Wuppertal \\ Gaussstr. 20, D-42119 Wuppertal, Germany \\ ${ }^{c}$ Department of Mathematics, Bergische Universität Wuppertal \\ Gaussstr. 20, D-42119 Wuppertal, Germany
}

\begin{abstract}
We present in detail a technique to extract the potential between a static quark and anti-quark pair from Wilson loops measured on dynamical configurations. This technique is based on HYP smearing and leads to an exponential improvement of the noise-to-signal ratio of Wilson loops. We explain why the correct continuum potential is obtained and show numerical evidence that the cut-off effects are small. We present precise results for the non-perturbative potential. As applications, we determine the scale $r_{0} / a$ and study the shape of the static potential in the range of distances around $r_{0}$, where it can be compared with phenomenological potential models.
\end{abstract}




\section{Introduction}

The potential $V(r)$ between a static (infinitely massive) quark and anti-quark pair separated by distance $r$ can be computed from lattice quantum chromodynamics (QCD). It is extracted from the expectation values of Wilson loops, which are traces of products of links along rectangular paths extending in Euclidean time and one spatial direction. In this article we consider only on-axis Wilson loops but off-axis (non-planar) Wilson loops can also be used. Alternatively the static potential can be extracted from the correlator of two Polyakov loops. Due to confinement, the signal of Wilson loops falls exponentially with the area of the loop (until string breaking sets in) but their variance is approximately constant. In the statistical average of standard Monte Carlo lattice simulations, the signal of Wilson loops is the result of strong cancellations between positive and negative contributions. This leads to an exponentially growing noise-to-signal ratio which prevents the calculation of the potential at large distances.

In pure gauge theory this problem has a cure. An exponential suppression of the statistical noise of Wilson loops can be achieved by the multi-hit (or one-link) method [1] and much further by the multilevel algorithm [2]. These algorithms are not applicable in presence of dynamical fermions due to the non-locality of the effective gauge action when the logarithm of the fermion determinant is included. In [3] a smearing technique called hypercubic (HYP) smearing was introduced which can also be used to measure Wilson loops in the presence of dynamical fermions [4]. In pure gauge theory it was demonstrated in [5] that the use of HYP smeared links leads to a determination of the static potential comparable in precision to the multi-hit method. In [6] a new action for static quarks was proposed, which uses HYP smeared links in the time covariant derivative of the EichtenHill action. This leads to an exponential reduction (compared with using the Eichten-Hill action) of the noise-to-signal ratio for heavy-light correlation functions. This effect is due to the fact that HYP smearing in the static action reduces the coefficient of the divergent part of the self-energy of a static quark [7,8].

The interest in the determination of the static potential $V(r)$ through lattice simulations is twofold. On the one side, there is the possibility to set the scale (i.e., determine the lattice spacing) through the scale $r_{0}$ introduced in [9]. The latter is defined from the static force $F(r)=V^{\prime}(r)$ as the solution of

$$
\left.r^{2} F(r)\right|_{r=r_{0}}=1.65 \text {. }
$$

A physical value for the scale $r_{0} \approx(0.45 \ldots 0.5)$ fm can only be determined through phenomenological potential models. It is desirable for an absolute determination of the lattice spacing to use a quantity which is directly accessible from experiment 
and replace $r_{0}$ by a quantity like a hadron mass or decay constant. But still, $r_{0}$ is very useful for a relative scale setting.

On the other side, the static potential is an interesting observable by itself for phenomenology (see the conclusions) and to study the structure of gauge theories [10 13]. It exhibits clear effects of dynamical fermions, such as string breaking [14, see the latest study in QCD [15] and high precision studies with multilevel algorithms in other models [16]. In order to study the potential at the distances where the string breaks, operators which dominantly create static-light meson pairs have to be included in addition to the Wilson loops and we plan to do so in the future. In this article we will concentrate on the determination of the static potential at distances smaller than the string breaking distance $r_{\mathrm{b}} \approx 3 r_{0}$ [17. We will study the quantity

$$
c(r)=\frac{1}{2} r^{3} F^{\prime}(r) .
$$

It is a physical, renormalized quantity, which can be used to define a running coupling. In [18] $c(r)$ has been determined with high precision in pure gauge theory using a multilevel technique. We will compute it in this article for QCD with $N_{\mathrm{f}}=2$ flavors of quarks.

In section two we will describe our techniques to extract the static potential from HYP smeared Wilson loops. We explain why this procedure leads to a determination of the continuum static potential up to $\mathcal{O}\left(a^{2}\right)$ lattice artifacts, which appear to be small. In section three we present our results for the static potential, the scale $r_{0} / a$ and the quantity $c(r)$ determined on a configuration ensemble generated with Wilson gauge action and $N_{\mathrm{f}}=2$ flavors of $\mathcal{O}(a)$ improved Wilson quarks at $\beta=5.3$. The quark mass corresponds to a pseudoscalar mass value close to $r_{0} m_{\mathrm{PS}}=1$ and we get a value $r_{0} / a=6.75(6)$.

\section{Techniques}

\subsection{Static potential with HYP smearing}

We measure $r / a \times T / a$ on-axis Wilson loops $W(r, T)$ on gauge configurations generated with $N_{\mathrm{f}}=2$ dynamical fermions. The technique is based on HYP smearing and was introduced in [3]. Before measuring the Wilson loops, we replace all the gauge links by HYP-smeared ones. We consider two choices of the HYPsmearing parameters: one is

$$
\alpha_{1}=0.75, \quad \alpha_{2}=0.6, \quad \alpha_{3}=0.3,
$$



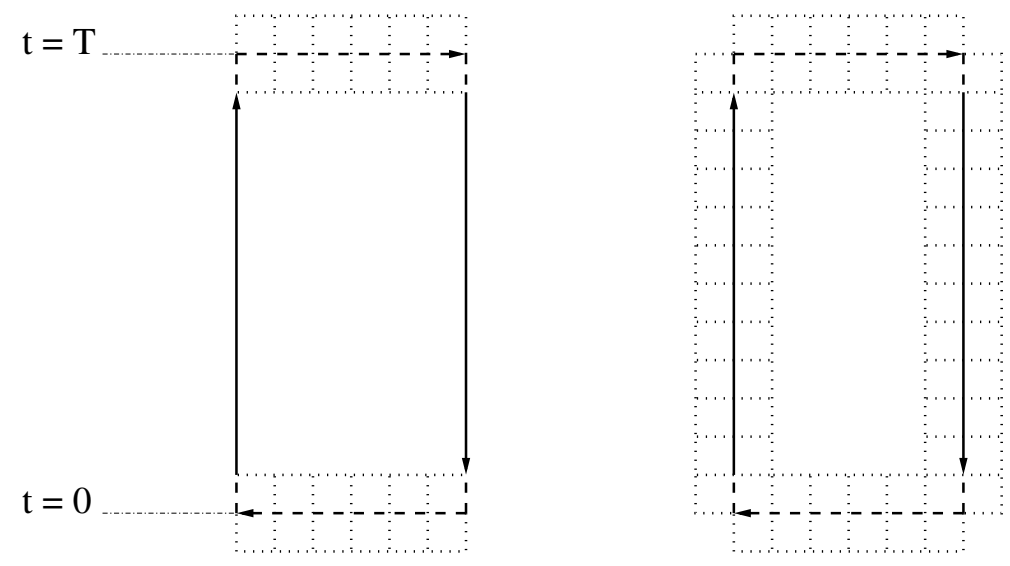

Figure 1: Schematic representation of the measurement of Wilson loops. In the first step (left figure) only the spatial Wilson lines are HYP-smeared: this corresponds to the definition of an operator $\hat{O}^{\dagger}$ that creates a $|Q \bar{Q}(r)\rangle$ state. In the second step (right figure) the temporal Wilson lines are HYP-smeared: this corresponds to the choice of the static quark action (and a modification of the operator $\hat{O}$ ).

which we refer to as HYP, and the other is

$$
\alpha_{1}=1.0, \quad \alpha_{2}=1.0, \quad \alpha_{3}=0.5,
$$

which we refer to as HYP2. We adopt the approximate projection onto $S U(3)$ as described in [7] and always use Eq. (2.24) and four iterations of Eq. (2.25) in [7].

In the following we show that this procedure leads to a determination of the potential between quark and anti-quark sources that agrees with the continuum potential up to $\mathcal{O}\left(a^{2}\right)$ effects (after renormalization). The ingredients in this demonstration are the selfadjoint positive transfer matrix of the lattice gauge theory with Wilson fermions and Wilson plaquette action (rigorously proven [19]) as well as the existence and universality of the continuum limit of the lattice theory with a static quark (lowest order of heavy quark effective theory [20]). The latter property is generally assumed and has been tested frequently (see [21] for a longer discussion).

For the purpose of showing Eq.(2.6), we split the HYP-smearing of the links used in building a Wilson loop into two steps, which are schematically represented in Fig. 1. In the first step we consider Wilson loops where only the space-like links are HYP smeared. The smearing involves links at time-slices $1=-a, 0, a$ and $t=T-a, T, T+a$ and corresponds in the Hamiltonian formalism to an operator $\hat{O}^{\dagger}$ and $\hat{O}$ that creates or annihilates a state $\left|\psi^{Q \bar{Q}}(r)\right\rangle$ consisting of a static quark

\footnotetext{
1 In total there are $N_{t}$ time-slices and periodic boundary conditions are imposed in all directions.
} 
and anti-quark pair at time-slices $t=a$ and $t=T-a$ respectively. The static sources are separated by a distance $r$ along one of the spatial directions. The path integral average of this Wilson loop can be written as a quantum mechanical expectation value

$$
\langle W(r, T)\rangle=\frac{\operatorname{Tr}\left\{\mathbb{T}_{0}^{N_{t}-T / a-2} \hat{O} \mathbb{T}_{q \bar{q}}(r)^{T / a-2} \hat{O}^{\dagger}\right\}}{\operatorname{Tr}\left\{\mathbb{T}_{0}^{N_{t}}\right\}},
$$

where $\mathbb{T}_{0}$ is the transfer matrix in the gauge-invariant (or zero charge) sector of the Hilbert space, $\mathbb{T}_{q \bar{q}}(r)$ the transfer matrix in the sector with a quark and an anti-quark source separated by $r$ and $\mathrm{Tr}$ is the operator trace in the Hilbert space. We denote the transfer matrix in the temporal gauge (where the timelike links are set to identity) by $\mathbb{T}_{\text {temp. }}$. The Hamiltonian $\mathbb{H}$ is defined through $a \mathbb{H}=-\ln \left\{\mathbb{T}_{\text {temp }}\right\}$. For the theory with Wilson quarks without a clover term $\mathbb{T}_{\text {temp }}$ has been constructed in [19]. The transfer matrix in a specific charge sector is obtained by multiplying $\mathbb{T}_{\text {temp }}$ with the projectors onto that charge sector. Note that the representation Eq. (2.3) differs from the usual one only in that the operators $\hat{O}$ represent fields in the path integral on three timeslices, not one. If written down explicitly in the form of [19] they involve integration kernels. But their explicit form is not relevant here. Using the spectral decomposition of the transfer matrices (see for example [22]) and taking the limit $N_{t} \rightarrow \infty$, Eq. (2.3) becomes

$$
\langle W(r, T)\rangle \stackrel{N_{t} \rightarrow \infty}{\sim} \sum_{n} c_{n} c_{n}^{*} \mathrm{e}^{-V_{n}(r)(T-2 a)},
$$

where $c_{n}=\left\langle n ; q \bar{q}\left|\hat{O}^{\dagger}\right| 0\right\rangle$ are the overlaps of states obtained by applying the operator $\hat{O}^{\dagger}$ to the vacuum $|0\rangle$ with the eigenstates $|n ; q \bar{q}\rangle$ of the Hamiltonian (with eigenvalues $E_{n}^{(q \bar{q})}(r)$ ) in the sector with a quark and an anti-quark source. In Eq. (2.4) we use $V_{n}(r)=E_{n}^{(q \bar{q})}(r)-E_{0}^{(0)}$, where $E_{0}^{(0)}$ is the vacuum energy. For example $V_{0}(r)$ is the static potential and $V_{1}(r)$ its first excitation.

In the second step we rewrite the Wilson loop as a path integral expectation value

$$
\begin{aligned}
\langle W(r, T)\rangle= & -\frac{1}{2}\left\langle\bar{\psi}_{h}(a, \overrightarrow{0}) P_{-}(a, \overrightarrow{0} ; a, r \hat{k}) \gamma_{5} \psi_{\bar{h}}(a, r \hat{k})\right. \\
& \left.\bar{\psi}_{\bar{h}}(T-a, r \hat{k}) P_{+}^{\dagger}(T-a, \overrightarrow{0} ; T-a, r \hat{k}) \gamma_{5} \psi_{h}(T-a, \overrightarrow{0})\right\rangle
\end{aligned}
$$

where $\psi_{h}, \bar{\psi}_{h}$ and $\psi_{\bar{h}}, \bar{\psi}_{\bar{h}}$ are the static quark and anti-quark fermion fields respectively 2 and $P_{ \pm}(t, \overrightarrow{0} ; t, r \hat{k})$ represents the gauge parallel transporter made from

${ }^{2}$ The prefactor $-\frac{1}{2}$ and the gamma-matrices are due to our choice of treating the static quark fields as 2-component static fermion fields, see for example [21]. 
a product of space-like HYP-links at time $t \pm a$ and temporal links at time $t \pm a$ in ₹-direction (dashed lines in Fig. 1).

After integration over the static fields, the static quark propagator generates the time-like links in the observable, cf. Eq. (2.4) in [7], and one recovers the Wilson loops. Different choices for the static quark action can be made, in particular we consider here the one where the covariant derivative in time involves HYP or HYP2 links [6]. It was shown in [23] that static potential differences (where the self energy is canceled) have $\mathcal{O}\left(a^{2}\right)$ leading lattice artifacts, essentially due to the automatic $\mathcal{O}(a)$ improvement of heavy quark effective theory [24]. This is true in the theory with dynamical fermions provided that they are $\mathcal{O}(a)$ improved 4 We therefore conclude

$$
V_{n}^{\mathrm{HYP} / \mathrm{HYP} 2}(r)-2 E_{\text {stat }}^{\mathrm{HYP} / \mathrm{HYP} 2}=V_{n}^{\text {continuum }}(r)-2 E_{\text {stat }}^{\text {continuum }}+\mathcal{O}\left(a^{2}\right),
$$

where for convenience we have subtracted $V(\infty)=2 E_{\text {stat }}$. Here $E_{\text {stat }}$ is the binding energy of a meson made of a static and a light dynamical quark.

In order to investigate the magnitude of the lattice artifacts we compare in the left panel of Fig. 2 the qq-coupling $\alpha_{\mathrm{qq}}(r)=r^{2} F(r) / C_{\mathrm{F}}$ for the HYP and HYP2 actions. The static force $F(r)$ is obtained from the static potential $F(r-a / 2)=$ $[V(r)-V(r-a)] / a$. Details about the extraction of the static potential from correlation functions of Wilson loops are presented in the next section. We use the dynamical configurations described in Section 3. The difference of the couplings is given, to leading order in the cut-off effects, by $a^{2} / r^{2} G(\Lambda r, m r)$. The function $G$ describes the $r$-dependence and quark-mass-dependence of the cut-off effects. The size of the cut-off effects is small but with our errors they are significantly different from zero for $r<r_{0}$. They happen to be most significant at $r \approx r_{0} / 2$.

In Section 2.3 we describe how improved observables can be defined such that these cut-off effects are eliminated at tree level and are substantially reduced nonperturbatively [23]. The right panel of Fig. 2 is the same as the left but using the improved definition of the force Eq. (2.14). The cut-off effects are visibly reduced. We emphasize that the figure is not sufficient to exclude cutoff effects which are independent of the choice of static action. Different lattice spacings are needed to study those.

Our choice of the static quark action and the smearing of the spatial links is with parameters HYP2 Eq. (2.2). It gives a static potential with a somewhat

\footnotetext{
${ }^{3}$ We smear also the temporal links contained in the definition of the parallel transporters $P_{ \pm}$in Eq. (2.5). This corresponds to a change in the definition of the operator $\hat{O}$ in Eq. (2.3) and has no consequences for the argument presented here.

${ }^{4}$ For Wilson fermions improvement is achieved by adding the clover term [25 27] or by using a twisted mass term [28] "at maximal twist" [29].
} 

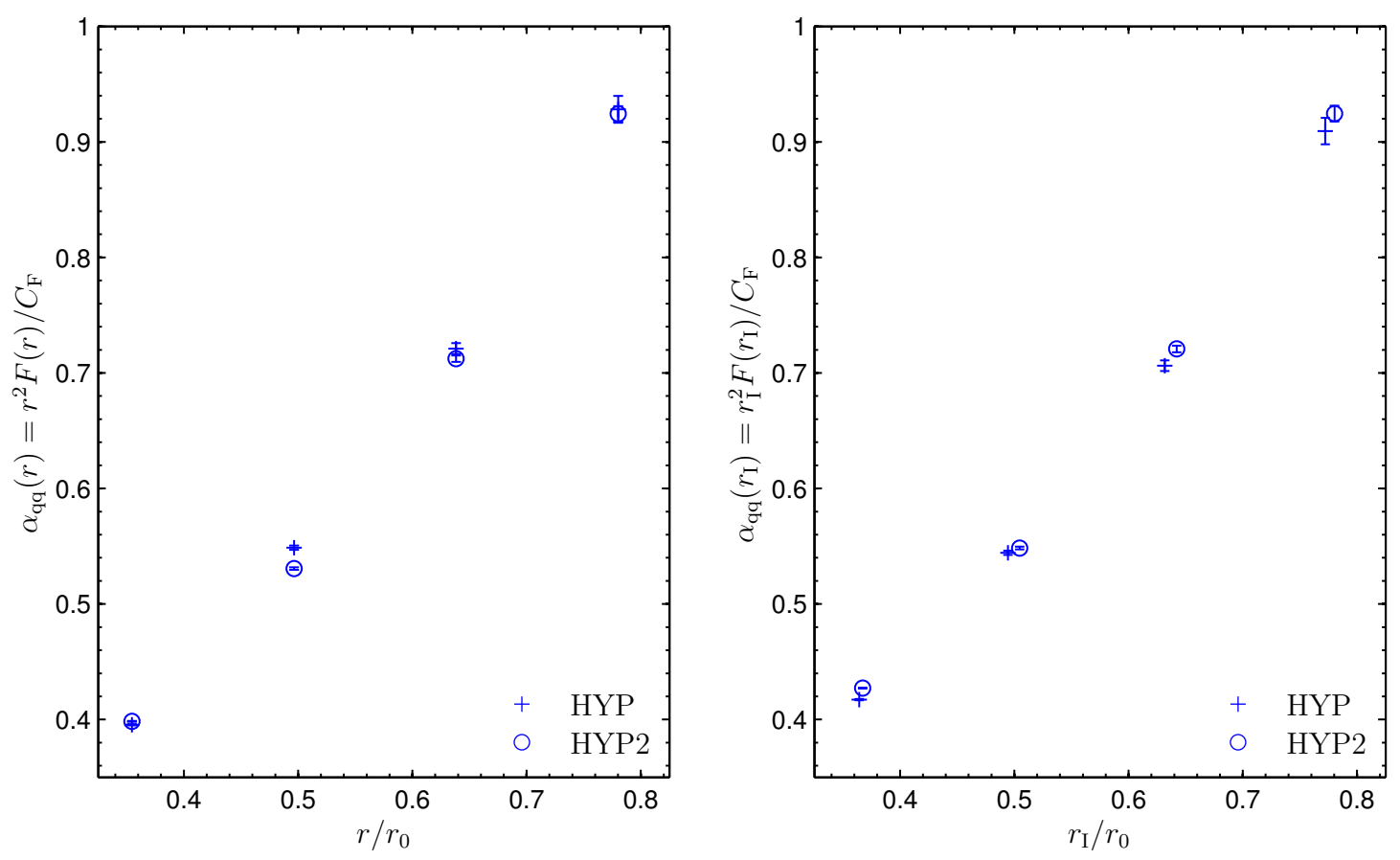

Figure 2: The qq-coupling $\alpha_{\mathrm{qq}}(r=1 / \mu)$ obtained from the static force $F(r-a / 2)=$ $[V(r)-V(r-a)] / a$ with two different choices of the static action (left panel). In Section 2.3 we give an improved definition of the force which is free of cutoff effects at tree level of perturbation theory (right panel).

better statistical precision than with parameters HYP. This can be understood in perturbation theory: the HYP2 parameters are such that they approximately minimize the one-loop coefficient of the $1 / a$ self-energy contribution of a static quark [7,8]. Our data show that this property remains true non-perturbatively: we find $V^{\mathrm{HYP} 2}-V^{\mathrm{HYP}} \approx-0.07 / a \approx 2\left(E_{\text {stat }}^{\mathrm{HYP}}-E_{\text {stat }}^{\mathrm{HYP}}\right)$. For the last statement we use the results for $E_{\text {stat }}$ of reference [30].

\subsection{Variational basis}

On the HYP2-smeared gauge link configurations $\{U(x, \mu)\}$, we measure a correlation matrix of on-axis Wilson loops at fixed spatial extension $r / a$ and temporal extension $T / a$ :

$$
C_{l m}(T)=\left\langle\operatorname{tr}\left\{P^{(l)}(0 ; r \hat{k}) P(r \hat{k} ; r \hat{k}+T \hat{0}) P^{(m) \dagger}(T \hat{0} ; r \hat{k}+T \hat{0}) P^{\dagger}(0, T \hat{0})\right\}\right\rangle
$$

where $P(x, y)$ represents the product of links connecting $y$ to $x$. Neglecting the superscripts on the spatial $P$ 's, Eq. (2.7) is equivalent to Eq. (2.5) after integrat- 
ing out the static fields. In the product of spatial links, the superscript $P^{(l)}$ means that the links $U_{l}(x, k)$ used in the product are obtained by applying the spatial smearing $\mathcal{S}_{\text {SHYP }}$ operator $n_{l}$ times

$$
U_{l}(x, k)=\left(\mathcal{S}_{\mathrm{sHYP}}\right)^{n_{l}} U(x, k) .
$$

$\mathcal{S}_{\text {sHYP }}$ means smearing with only two levels of HYP blocking with staples restricted to spatial directions and therefore it needs two parameters, which we set to $\alpha_{2}=0.6$ and $\alpha_{3}=0.3$. In the argument of the previous section the fat parallel transporters $P^{(l)}$ correspond to operators $\hat{O}_{l}$ implementing trial states $\left|\psi_{l}^{Q \bar{Q}}(r)\right\rangle=\hat{O}_{l}^{\dagger}|0\rangle$. In [31] a formula for suitable smearing parameters $n_{l}$ is given in the case of APE smearing. In order to choose $n_{l}$ for spatial HYP smearing, we use the result of [32], that the mean squared extension of APE smearing is approximately $\alpha_{\mathrm{APE}} n_{l, \mathrm{APE}} a^{2} / 3$, and require that this is equal to $n_{l} a^{2}$ for HYP smearing. We get an approximate formula for a good range of HYP smearing levels

$$
n_{l} \approx \frac{l}{12}\left(\frac{r_{0}}{a}\right)^{2}
$$

For our data on the configuration ensemble E5g (see Section 3) we have computed a large correlation matrix using smearing levels $n_{0,1,2,3,5}$. We find that this basis can be reduced to an optimal subset of $M=3$ levels

$$
n_{2}=8, \quad n_{3}=12, \quad n_{5}=20, \quad \text { at } \beta=5.3 .
$$

The higher smearing levels improve the determination of the energy levels.

We use the generalized eigenvalue method [31,33 35] to extract the ground state potential as follows. We first solve the generalized eigenvalue problem

$$
C(t) \psi_{\alpha}=\lambda_{\alpha}\left(t, t_{0}\right) C\left(t_{0}\right) \psi_{\alpha}
$$

Then we perform a fit to

$$
E_{\alpha}\left(t+\frac{a}{2}, t_{0}\right) \equiv \ln \left(\lambda_{\alpha}\left(t, t_{0}\right) / \lambda_{\alpha}\left(t+a, t_{0}\right)\right)=E_{\alpha}+\beta_{\alpha} \mathrm{e}^{-\left(E_{M}-E_{\alpha}\right)\left(t+\frac{a}{2}\right)},
$$

with fit parameters $E_{\alpha}, \beta_{\alpha}$ and $E_{M}$, simultaneously for $\alpha=0,1$ ( $\alpha=0$ corresponds to the ground state, $\alpha=1$ to the first excited state), $t_{0} / a=2,3,4$ and $t_{0}+a \leq t \leq 2 t_{0}$ (the latter constraint is necessary for eq.(2.12) to hold [35] ), i.e., we have 18 data points $E_{\alpha}\left(t+\frac{a}{2}, t_{0}\right)$ for 5 fit parameters.

The values of the ground state potential $V(r)$ as a function of $r$ are determined from a plateau average of the corresponding effective masses $E_{0}\left(t, t_{0}\right)$ starting at 


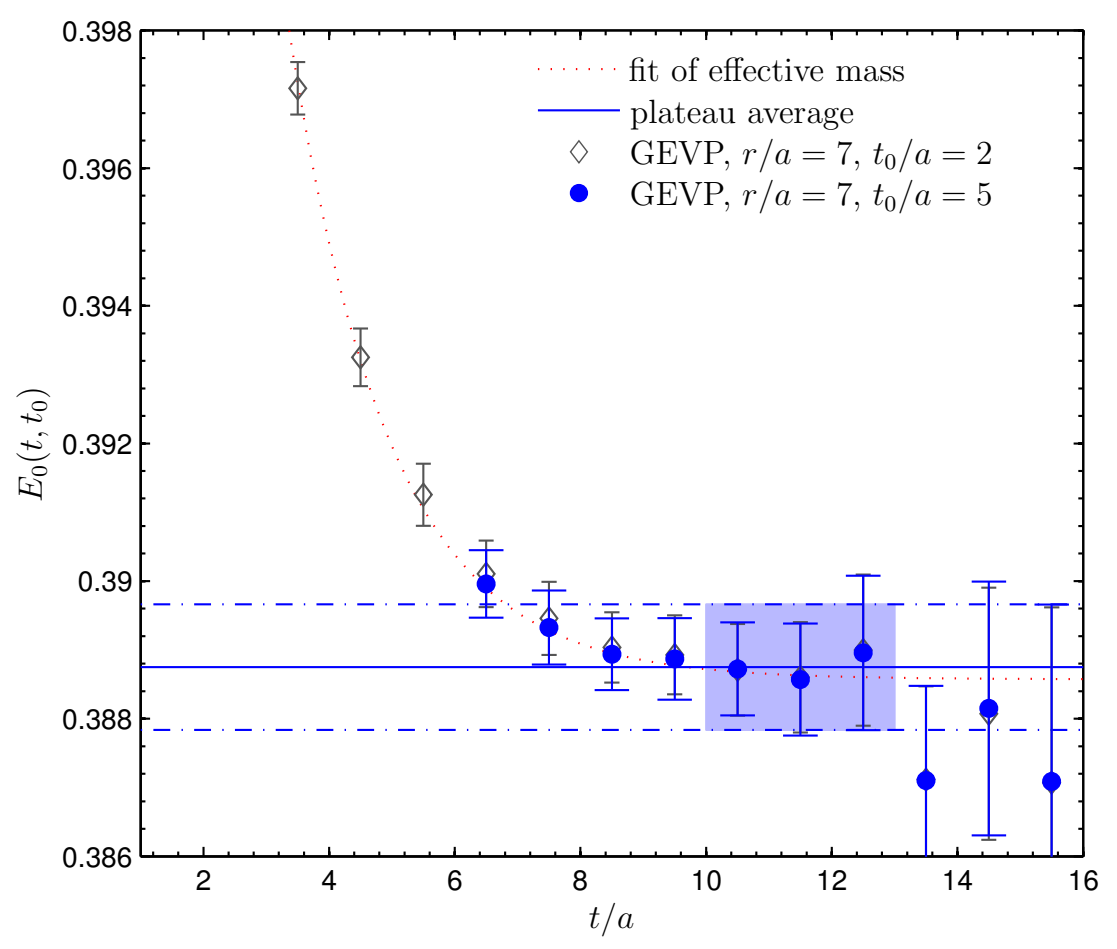

Figure 3: Effective masses $E_{0}\left(t, t_{0}\right)$ (filled blue circles for $t_{0} / a=5$, empty black diamonds for $t_{0} / a=2$ ) for the ground state potential at $r=7 a$. The red dotted line is the fit Eq. (2.12). The blue line is the plateau average from the points in the blue shaded area (the blue dashed-dotted lines are the plateau errors).

the value $t=2 t_{0}+\frac{a}{2}$, where the fixed value $t_{0}$ is determined by the requirement that

$$
\sigma_{\text {sys }}\left(E_{0}\left(2 t_{0}+\frac{a}{2}, t_{0}\right)\right) \equiv \beta_{0} \mathrm{e}^{-\left(E_{M}-E_{0}\right)\left(2 t_{0}+\frac{a}{2}\right)} \lesssim \frac{1}{4} \sigma_{\text {stat }}\left(E_{0}\left(2 t_{0}+\frac{a}{2}, t_{0}\right)\right),
$$

where $\sigma_{\text {sys }}(\cdot)$ and $\sigma_{\text {stat }}(\cdot)$ denote the systematic and statistical error respectively. For our data, Eq. (2.13) is satisfied for $t_{0} / a=5$ for all values of $r$. The plateau average is stopped before the time, when either the difference of the effective mass with the one at $t=2 t_{0}+\frac{a}{2}$ is larger than the statistical error of the latter or the statistical error of the effective mass is larger than twice the one of the effective mass at $t=2 t_{0}+\frac{a}{2}$. The effective masses $E_{0}\left(t, t_{0}\right)$ (filled blue circles) together with the fit Eq. (2.12) (red dotted line) and the plateau average (blue line with error band marked by blue dashed-dotted lines) for $r=7 a \approx r_{0}$ are shown in Fig. 3 . The plateau average comprises three points at $t / a=10.5,11.5,12.5$. The error of the plateau average is the sum of the statistical and the systematic errors, with the latter being given by the left-hand side of Eq. (2.13). For comparison, we also plot in Fig. 3 the effective masses obtained using $t_{0} / a=2$ (empty black diamonds). 


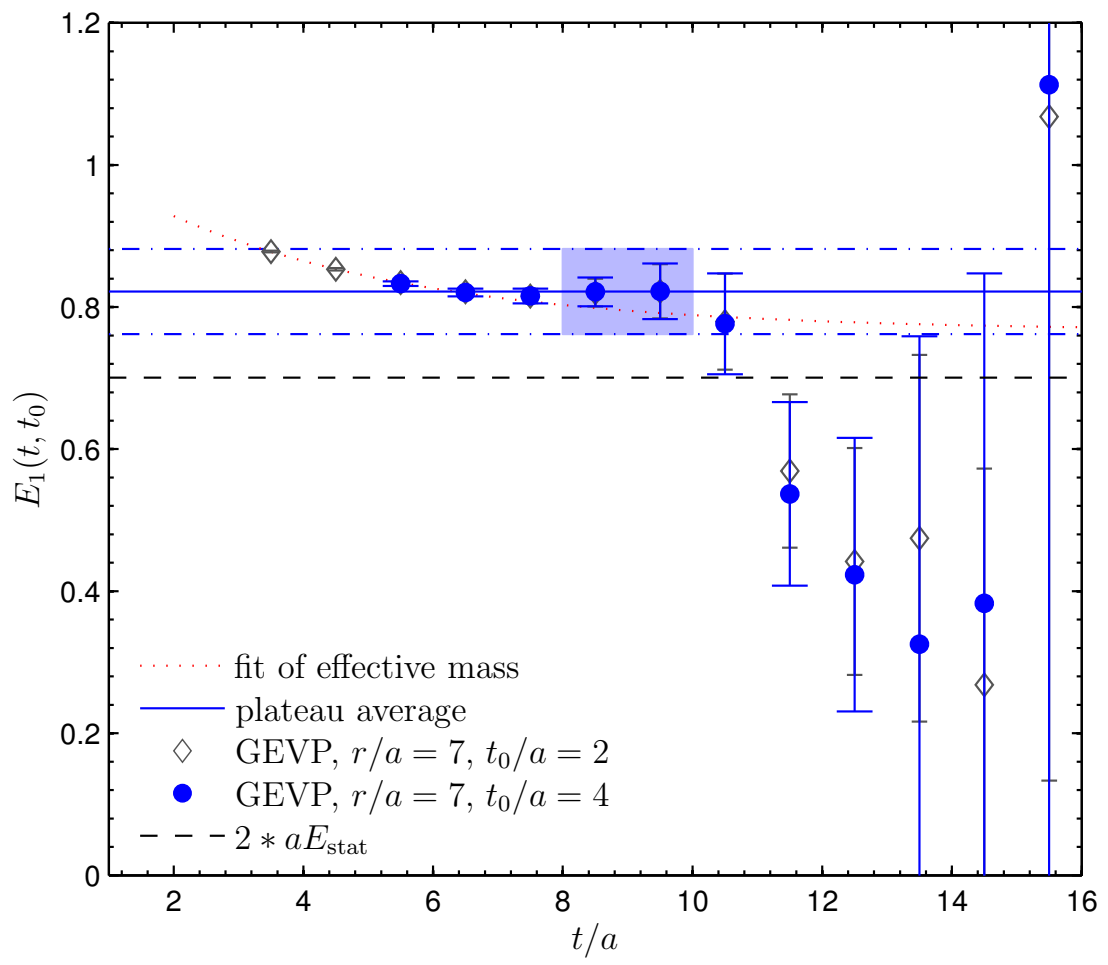

Figure 4: Effective masses $E_{1}\left(t, t_{0}\right)$ (filled blue circles for $t_{0} / a=4$, empty black diamonds for $t_{0} / a=2$ ) for the first excited state potential at $r=7 a$. The dashed black line represents the value $2 a E_{\text {stat }}$, the meaning of the other curves is as explained in Fig. 3 ,

They are part of the data set fitted using Eq. (2.12). At the times when they are both defined, the effective masses for $t_{0} / a=5$ and $t_{0} / a=2$ agree with each other, which is somewhat surprising since non-leading corrections certainly have a dependence on $t_{0}$.

In principle the excited potentials can be determined in the same way. However, the analysis is complicated by the dynamics of string breaking. From model studies [36 38] as well as from [15], we know that an extraction of the potentials requires the inclusion of operators which dominantly create static-light meson pairs in addition to the string-like operators we use here. Only then does the ground state at large distances $r>r_{\mathrm{b}}$ contribute significantly to the spectral decomposition of the correlation function matrices at the accessible time separations (cf. [39]). While we are not concerned here with this string breaking region, it is known [15, 38 that for $r<r_{\mathrm{b}}$ the first excited state is an (approximate) mesonanti-meson state at $V_{1} \approx 2 E_{\text {stat }}$. This state is not well seen in our computation which does not include the meson pair operators. In Fig. [4 we show the effective masses $E_{1}$ for $r=7 a \approx r_{0}$. The dashed black line represents $2 a E_{\text {stat }}=0.7007(14)$, 
the meaning of the other curves is as explained for Fig. 3. Although the effective masses seem to form a plateau at times $t=8.5 a$ and $t=9.5 a$, they drop at values $t \geq 10.5 a$, but the statistical errors are too large in this region to determine an energy level. For $r<r_{0}$ we see plateaus for $E_{1}$ which are compatible with $2 a E_{\text {stat }}$.

This deficiency of our variational basis also affects the estimate of the ground state potential, but here the only concern is the description of the corrections to the asymptotic plateau of the form $\beta_{0} \mathrm{e}^{-\left(E_{M}-E_{0}\right) t}$. These enter the final numbers and errors only to estimate where we start the plateau average such that excited state contaminations are small compared to our statistical error. A very precise determination of $E_{M}$ or in general of the excited states is not necessary for this purpose. Furthermore, the effective mass figures show that our plateau selection is rather conservative; the extracted ground state potential is reliable within the cited errors.

\subsection{Tree level improved force}

In order to determine the scale $r_{0}$ [9] from Eq. (1.1) we will need the static force $F(r)$. An improved definition of the force on the lattice is $[9,23,31]$

$$
F\left(r_{\mathrm{I}}\right)=[V(r)-V(r-a)] / a
$$

where $r_{\mathrm{I}}=r-a / 2+\mathcal{O}\left(a^{2}\right)$ is chosen such that at tree level in perturbation theory [40] one has

$$
F_{\text {tree }}\left(r_{\mathrm{I}}\right)=C_{\mathrm{F}} \frac{g_{0}^{2}}{4 \pi r_{\mathrm{I}}^{2}},
$$

where $C_{\mathrm{F}}=4 / 3$ for gauge group $S U(3)$. The formula for $r_{\mathrm{I}}$ depends on the static quark action and it is given in Appendix A for HYP actions.

An improved lattice definition of the quantity $c(r)$ in Eq. (1.2) is given by [18]

$$
c(\tilde{r})=\frac{1}{2} \tilde{r}^{3}[V(r+a)+V(r-a)-2 V(r)] / a^{2},
$$

where $\tilde{r}=r+\mathcal{O}\left(a^{2}\right)$ is chosen such that

$$
c_{\text {tree }}(\tilde{r})=-C_{\mathrm{F}} \frac{g_{0}^{2}}{4 \pi} .
$$

The formula for $\tilde{r}$ depends on the static quark action and it is given in Appendix A for the HYP actions. In Appendix B we give the 4-loop beta function for the coupling $\alpha_{\mathrm{c}}=-c(r) / C_{\mathrm{F}}$ which we will use to generate perturbative curves for $c(r)$ to be compared with the lattice data. 


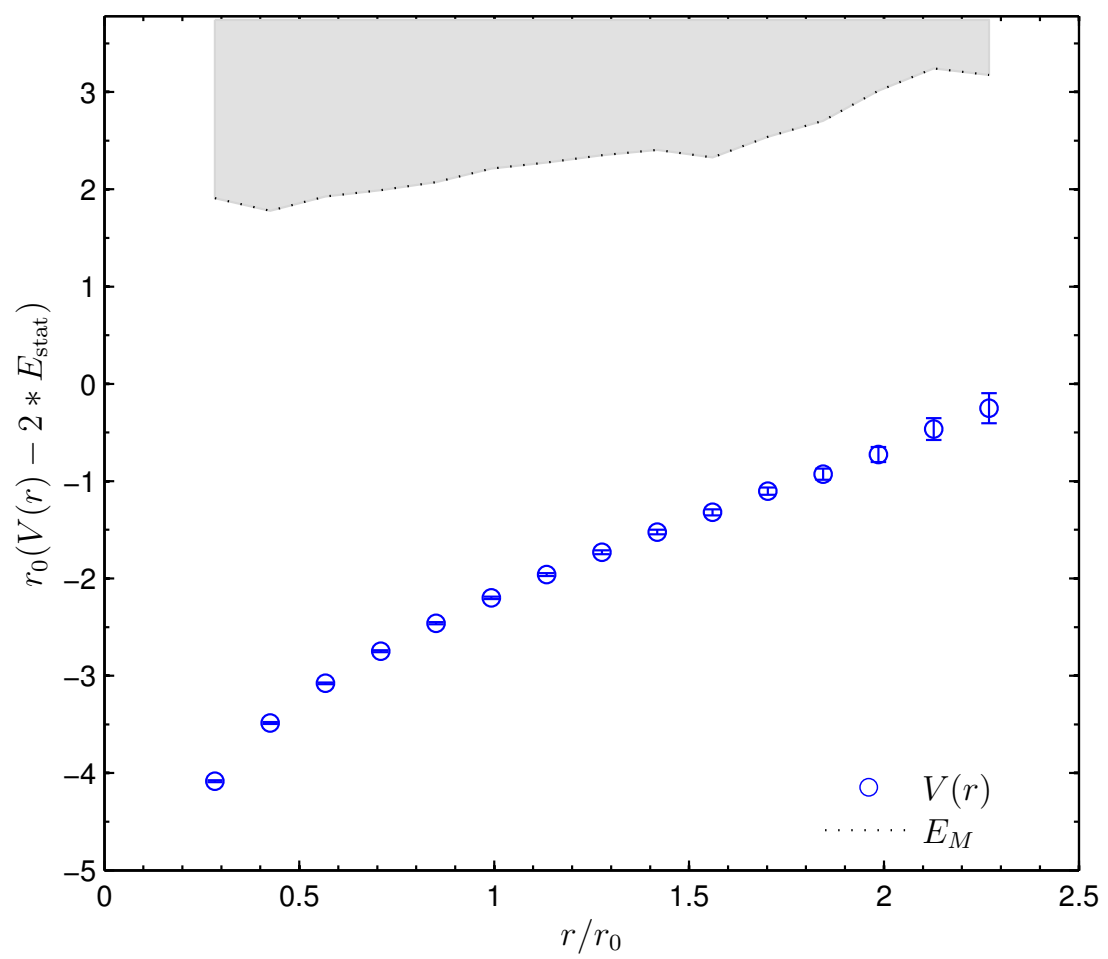

Figure 5: Ground state potential $V(r)$ (circles). The shaded area marks energy states larger than $E_{M}$ (here $M=3$ ) determined from Eq. (2.12).

\section{Results}

We compute the static potential on the lattice ensemble E5g generated by the CLS ("Coordinated Lattice Simulations") project5 at $\beta=5.3, \kappa=0.13625$ with geometry $64 \times 32^{3}$ and periodic boundary conditions for all fields apart from anti-periodic boundary conditions for the fermions in time. The value of the pseudo-scalar mass is $a m_{\mathrm{PS}}=0.15$. The algorithm used in CLS is the deflation accelerated DD-HMC algorithm [41,42]. The trajectory length is $\tau=4$ and we separate the measurements of Wilson loops by 4 trajectories. Given the block size $8^{4}$, the active links represent $37 \%$ of all links. Hence the separation in molecular dynamics time between measurements is approximately 6 units (when all links are changed). We have a statistics of about 1000 measurements.

In Fig. 5 we show the ground state potential $V$, and for illustration the rough estimate of the excitation $E_{M}$ (here $M=3$ ) in Eq. (2.12). In order to get renormalized quantities we subtract twice the binding energy $E_{\text {stat }}$ of a meson made of a static and a light dynamical quark. Everything is made dimensionless

\footnotetext{
${ }^{5}$ https://twiki.cern.ch/twiki/bin/view/CLS/WebHome
} 


\begin{tabular}{clcl}
\hline & & & \\
$r_{\mathrm{I}} / a$ & $a^{2} F\left(r_{\mathrm{I}}\right)$ & $\tilde{r} / a$ & $c(\tilde{r})$ \\
\hline & & & \\
3.55805 & $0.05776(12)$ & 4.046306 & $-0.3596(41)$ \\
4.52674 & $0.04690(19)$ & 5.026094 & $-0.391(15)$ \\
5.50073 & $0.04074(35)$ & 5.999703 & $-0.405(41)$ \\
6.48362 & $0.03699(48)$ & 6.977869 & $-0.519(99)$ \\
7.47397 & $0.03393(75)$ & 7.917429 & $-0.35(22)$ \\
8.46922 & $0.0325(12)$ & & \\
9.46734 & $0.0295(13)$ & & \\
10.4670 & $0.0287(19)$ & & \\
11.4676 & $0.0310(25)$ & & \\
12.4685 & $0.0248(53)$ & & \\
13.4697 & $0.0285(48)$ & & \\
14.4709 & $0.0373(75)$ & & \\
15.4721 & $0.030(11)$ & & \\
\hline
\end{tabular}

Table 1: The values of the force $F(r)$ in lattice units and the physical quantity $c(r)$ at the accessible improved distances $r_{\mathrm{I}}$ and $\tilde{r}$ respectively. We do not include values that require the potential $V(r=2 a)$, since it may be affected by large cut-off effects.

by appropriate powers of $r_{0}$ extrapolated to the chiral limit, see below. The first excited state potential is not shown due to the difficulties described above.

The range of string breaking is not yet reached. We can estimate it from the condition $V\left(r_{\mathrm{b}}\right)=2 E_{\text {stat }}$ to be

$$
2.4 \leq\left.\frac{r_{\mathrm{b}}}{r_{0}}\right|_{r_{0} m_{\mathrm{PS}}=1.0} \leq 2.6 .
$$

For comparison, in [15] $r_{\mathrm{b}} / r_{0} \approx 2.5$ was found at a larger quark mass corresponding to $r_{0} m_{\mathrm{PS}}=1.7$, albeit in the theory without $\mathcal{O}(a)$ improvement.

The scale $r_{0}$ is defined from the condition Eq. (1.1). The static force is computed from Eq. (2.14) using the improved distance $r_{\mathrm{I}}$ in Eq. (A.1). In Table 1 we list the values of the force in lattice units. We do not include the force at $r_{\mathrm{I}} / a=2.58875$ because it requires the potential at distance $r=2 a$, which may be affected by relatively large cut-off effects. We determine the solution of Eq. (1.1) by interpolation of the force $F$, using a 2-point interpolation $F(r)=f_{0}+f_{2} / r^{2}$ and a 3 -point interpolation adding a $f_{4} / r^{4}$ term to control the systematic error (it is found to be negligible). We obtain

$$
\left.\frac{r_{0}}{a}\right|_{a m_{\mathrm{PS}}=0.15}=6.747(59) \quad(\beta=5.3) .
$$




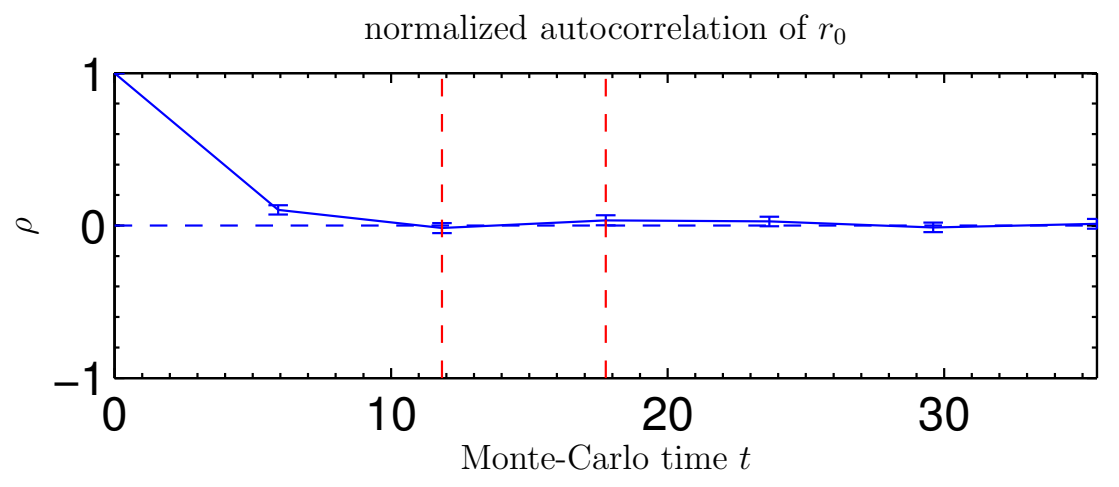

upper and lower bound of $\tau_{\text {int }}$ with statistical errors

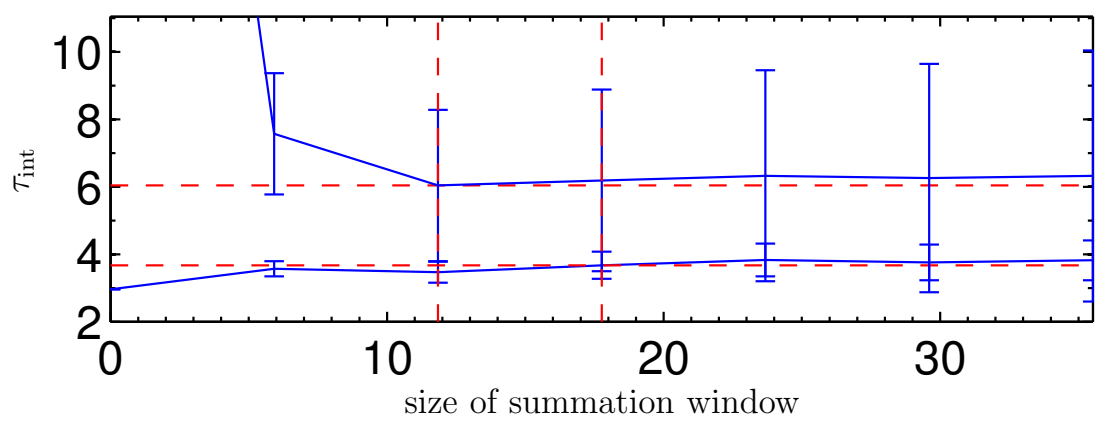

Figure 6: Auto-correlation function $\rho(t)$ and integrated auto-correlation time $\tau_{\text {int }}$ of $r_{0}$. The Monte-Carlo time is in units of molecular dynamics time.

The error is determined by taking the upper bound $\tau_{\text {int }}=6$ (see below) and neglecting the systematic error (due to excited state contributions), which is much less than the statistical one (due to condition Eq. (2.13)). In [43] we presented a preliminary value $r_{0} / a=7.05(3)$ extrapolated to the chiral limit. Throughout this article we use this value (without errors) for the purpose of plotting dimensionless quantities.

In 44 it was shown that the auto-correlation time of the topological charge suffers from critical slowing down proportional to $a^{-5}$ in the present range of lattice spacings. However, in the same reference it was shown that Wilson loops are affected by a much milder critical slowing down, implying that their coupling to the slow modes of the Monte Carlo simulation is small. A method for correcting the error analysis, by adding a tail to the auto-correlation function that takes into account the coupling to slow modes, was presented in [44]. We use this method in our data analysis and we set $\tau_{\exp }=39$ in molecular dynamics unit: 6 from Table 4 of [44]. In Fig. 6 we show the auto-correlation function $\rho(t)$ and the integrated

\footnotetext{
${ }^{6}$ In these units, the DD-HMC molecular dynamics time is multiplied by the ratio of active links, which in our case is $37 \%$.
} 


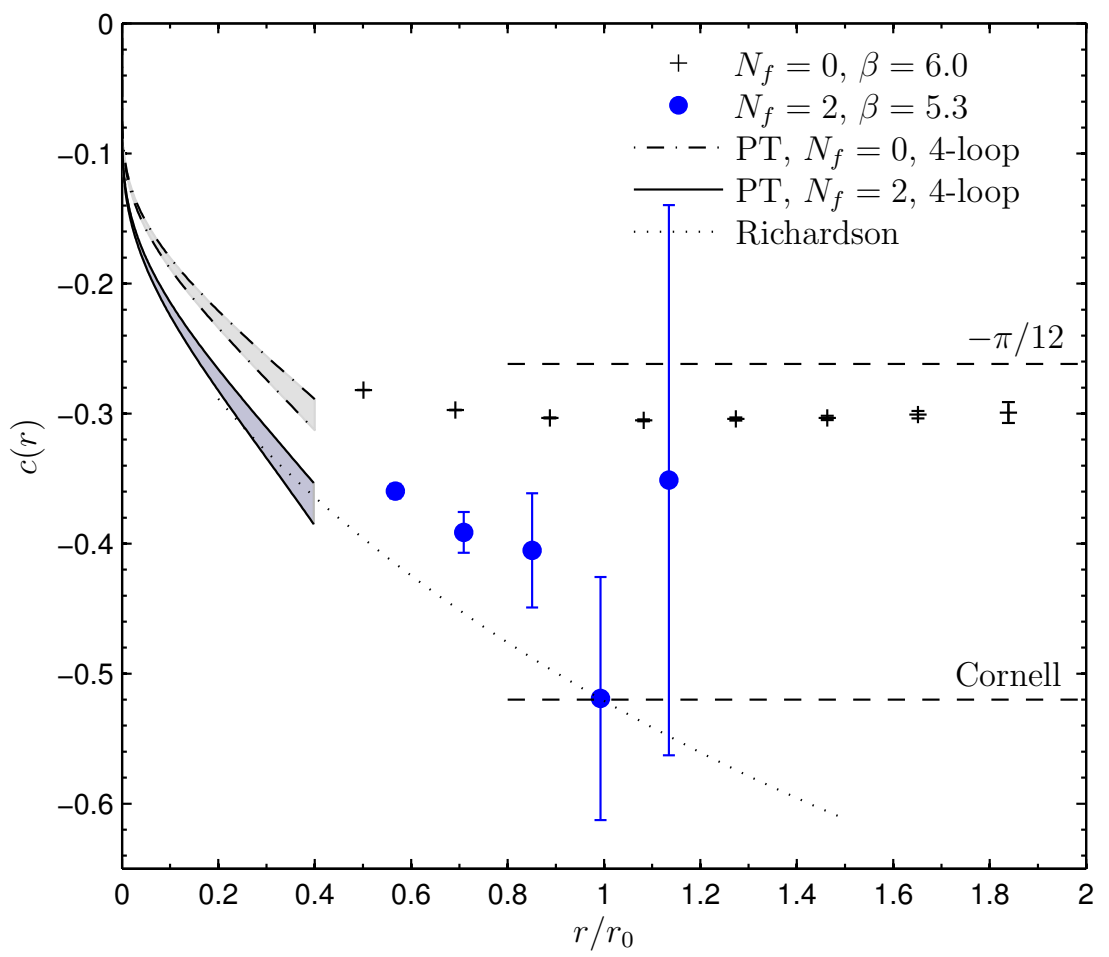

Figure 7: The physical quantity $c(r)$ in Eq. (1.2). Comparison of $N_{\mathrm{f}}=2$ (circles) with $N_{\mathrm{f}}=0$ (pluses) Monte Carlo data (for $N_{\mathrm{f}}=0$ taken from [18]) and perturbation theory. Also the value $c=-0.52$ in the Cornell [46] potential and the curve (dotted) derived from the Richardson [47] potential are plotted.

autocorrelation time $\tau_{\text {int }}$ of $r_{0}$, determined with the program 7 of [44] implementing the method of [44,45]. The vertical dashed lines in the plots mark the applied summation windows, the lower one is used when we add the tail due to the slow modes, while the larger one comes from using the method of [45]. Adding to the summed autocorrelation function the correction due to the slow modes leads to the upper curve and upper bound on $\tau_{\text {int }}$, which we take for all quantities in our analysis. The lower curve corresponds to $\tau_{\text {int }}$ determined from [45]. For $r_{0}$ we get an upper bound $\tau_{\text {int }}=6$ which is a factor 6.5 smaller than $\tau_{\text {exp }}$, but a factor 1.5 larger than without accounting for effects of undetected slow modes (lower bound).

In Fig. 7 we plot our result for the physical quantity $c(r)$ computed from Eq. (2.16) using the improved distance $\tilde{r}$ in Eq. (A.5). The numbers are given in Table 1. In order to compare our $N_{\mathrm{f}}=2$ results (circles), we plot them together with the $N_{\mathrm{f}}=0$ data (pluses) of [18] and with the perturbative curves obtained

\footnotetext{
${ }^{7}$ http://www-zeuthen.desy.de/alpha/
} 
using the 4-loop beta function (continued line for $N_{\mathrm{f}}=2$, dashed-dotted line for $\left.N_{\mathrm{f}}=0\right)$. The perturbative formula for $c(r)$ is presented in Appendix B and we used the preliminary updated value of the $\Lambda$ parameter presented in [43]. The spread of the perturbative curve reflects the uncertainty of the $\Lambda$ parameter. For a comparison with our Monte Carlo data, it is legitimate to plot the perturbative curve of $c(r)$ in massless perturbation theory, since quark mass corrections are of order $\alpha^{2} \times\left(m_{q} r\right)^{2}$ and are expected to be negligible at our small quark mass. The distances in Fig. 7 are normalized by $r_{0}$ extrapolated to the chiral limit. As the perturbative curves already indicate, the value of $c$ for $N_{\mathrm{f}}=2$ is found to be lower than for $N_{\mathrm{f}}=0$. In pure gauge theory, $c(r)$ starts approaching the asymptotic value $c(\infty)=-\pi / 12$ with corrections of order $1 / r$ as predicted from the effective bosonic string theory [48,49]. Our data for $N_{\mathrm{f}}=2$ have quite large errors when $r / r_{0} \geq 1$. We compare them with the value $c=-0.52$ that it takes in the phenomenological Cornell potential [46] and with the curve obtained from the Richardson potential [47]. Our data seem to follow the Richardson curve for $r \lesssim r_{0}$ quite closely. It is not yet possible to tell whether there is a plateau region around or above $r_{0}$ before string breaking sets in. We will return to this quantity in our future studies.

The comparison to the purely perturbative curve shows qualitative agreement. A meaningful quantitative comparison requires a careful study of lattice artifacts which may be quite noticeable in the region of small $r$, where perturbation theory applies. Indeed perturbation theory by itself suggests that at least $r \leq \frac{1}{2} r_{0}$ is necessary [50], in particular when the new 4-loop beta function is taken into account as discussed in appendix B.

\section{Conclusions}

We have presented a detailed analysis of the static potential defined by the HYP2 action for the static quarks. Fig. 5 and Fig. 7 show the quality of our data. Judged by a comparison of HYP and HYP2, cut-off effects in the potential appear to be small. The scale $r_{0} / a$ can be determined with precision better than $1 \%$. We observe large effects due to dynamical fermions in the quantity $c(r)$ defined in Eq. (1.2).

As can be seen in Table 1 the error on the force grows faster with the distance $r$ as compared to the pure gauge case (see Table 2 of [18]). This effect is amplified by $r^{3}$ for the quantity $c(r)$. It remains to be seen whether the inclusion of fermionic correlators in the variational basis will lead to an improvement due to a larger overlap with the ground state and the resulting earlier start of a plateau.

A precise study of the static potential is relevant for phenomenology in an 
indirect but important way. As reviewed in [51], there is an impressive effort to apply potential non-relativistic QCD (pNRQCD) [52] to the top - anti-top production in a future $e^{+} e^{-}$collider and to many other processes. This effective theory includes ultrasoft gluons and is treated perturbatively in the QCD coupling. While the potential of pNRQCD is not the same as the static potential, the two are intimately related; they differ only starting at NNNLO accuracy. It is hence very useful to understand where the perturbative approximation to the static potential can be trusted. Fig. 7 is a start for that, but a precise investigation requires the removal of lattice artifacts [23]. In the future we plan to work both on this connection to the perturbative regime of QCD and on the large distance, string breaking, region.

Acknowledgement. We are grateful to Nazario Tantalo for extensive checks of the Wilson loop measurements and to Stefan Schaefer for help in checking the HYP smearing. We thank Nikos Irges for discussions on the quantity $c(r)$ and Valentina Forini for discussions on the AdS/CFT correspondence. We further thank NIC and the Zuse Institute Berlin for allocating computing resources to this project. Part of the Wilson loop measurements were performed on the PCcluster of DESY, Zeuthen.

\section{A Improvement}

The tree level perturbative expression for the static potential, which is extracted from Wilson loops where the static quark line is HYP smeared, is given in [4,53]. From it we easily derive the formula for $r_{\mathrm{I}}$ defined from Eq. (2.15):

$$
\left(4 \pi r_{\mathrm{I}}^{2}\right)^{-1}=-\left[G_{\mathrm{HYP}}(r, 0,0)-G_{\mathrm{HYP}}(r-a, 0,0)\right] / a,
$$

with

$$
G_{\mathrm{HYP}}(\vec{r})=\frac{1}{a} \int_{-\pi}^{\pi} \frac{\mathrm{d}^{3} p}{(2 \pi)^{3}} \frac{\prod_{j=1}^{3} \cos \left(x_{j} p_{j} / a\right) \times f_{\mathrm{sm}}(p)}{\sum_{j=1}^{3} \hat{p}_{j}^{2}},
$$

where $\vec{r}=\left(x_{1}, x_{2}, x_{3}\right), \hat{p}_{j}=2 \sin \left(p_{j} / 2\right)$ and the smearing factor is

$$
\begin{aligned}
& f_{\mathrm{sm}}(p)=\left[1-\frac{\alpha_{1}}{6} \sum_{j=1}^{3} \hat{p}_{j}^{2} \Omega_{j 0}(p)\right]^{2} \\
& \Omega_{j 0}(p)=1+\alpha_{2}\left(1+\alpha_{3}\right)-\frac{\alpha_{2}}{4}\left(1+2 \alpha_{3}\right)\left(\hat{p}_{1}^{2}+\hat{p}_{2}^{2}+\hat{p}_{3}^{2}-\hat{p}_{j}^{2}\right)+\frac{\alpha_{2} \alpha_{3}}{4} \prod_{\tau \neq 0, j} \hat{p}_{\tau}^{2}
\end{aligned}
$$




\begin{tabular}{|c|c|c|c|c|}
\hline \multirow[t]{2}{*}{$r / a$} & \multicolumn{2}{|c|}{$r_{\mathrm{I}} / a$} & \multicolumn{2}{|c|}{$\tilde{r} / a$} \\
\hline & HYP & HYP2 & HYP & HYP2 \\
\hline 4 & 3.48560 & 3.55805 & 3.97292 & 4.04631 \\
\hline 5 & 4.45369 & 4.52674 & 4.93158 & 5.02609 \\
\hline 6 & 5.44414 & 5.50073 & 5.91700 & 5.99970 \\
\hline 7 & 6.44353 & 6.48362 & 6.91468 & 6.97787 \\
\hline 8 & 7.44614 & 7.47397 & 7.91743 & 7.96350 \\
\hline 9 & 8.44969 & 8.46922 & 8.92199 & 8.95537 \\
\hline 10 & 9.45331 & 9.46734 & 9.92696 & 9.95146 \\
\hline 11 & 10.4567 & 10.4670 & 10.9318 & 10.9501 \\
\hline 12 & 11.4598 & 11.4676 & 11.9362 & 11.9503 \\
\hline 13 & 12.4625 & 12.4685 & 12.9403 & 12.9512 \\
\hline 14 & 13.4649 & 13.4697 & 13.9440 & 13.9527 \\
\hline 15 & 14.4671 & 14.4709 & 14.9472 & 14.9543 \\
\hline 16 & 15.4690 & 15.4721 & 15.9502 & 15.9560 \\
\hline 17 & 16.4707 & 16.4733 & 16.9529 & 16.9576 \\
\hline 18 & 17.4723 & 17.4745 & 17.9553 & 17.9593 \\
\hline 19 & 18.4737 & 18.4755 & 18.9575 & 18.9609 \\
\hline 20 & 19.4750 & 19.4765 & 19.9595 & 19.9624 \\
\hline 21 & 20.4762 & 20.4775 & 20.9613 & 20.9638 \\
\hline 22 & 21.4772 & 21.4784 & 21.9630 & 21.9651 \\
\hline 23 & 22.4782 & 22.4792 & 22.9645 & 22.9664 \\
\hline 24 & 23.4791 & 23.4800 & 23.9659 & 23.9676 \\
\hline 25 & 24.4799 & 24.4807 & 24.9672 & 24.9687 \\
\hline 26 & 25.4807 & 25.4814 & 25.9685 & 25.9698 \\
\hline 27 & 26.4814 & 26.4820 & 26.9696 & 26.9708 \\
\hline 28 & 27.4820 & 27.4826 & 27.9706 & 27.9717 \\
\hline 29 & 28.4827 & 28.4831 & 28.9716 & 28.9726 \\
\hline 30 & 29.4832 & 29.4837 & 29.9726 & 29.9734 \\
\hline 31 & 30.4838 & 30.4842 & 30.9734 & 30.9742 \\
\hline 32 & 31.4843 & 31.4846 & 31.9742 & 31.9749 \\
\hline
\end{tabular}

Table 2: The values of the improved distances $r_{\mathrm{I}} / a$ Eq. (A.1) and $\tilde{r} / a$ Eq. (A.5) extrapolated to $L / a \rightarrow \infty$ for the case of HYP and HYP2 smearings. We show 6 significant digits for all values of $r / a$, where the last digit is rounded. 
$\left(f_{\mathrm{sm}}=1\right.$ for unsmeared static quark lines $)$.

The distance $\tilde{r}$ defined from Eq. (2.17) is given in the case of HYP smeared static quarks by

$$
\tilde{r}^{-3}=2 \pi\left[G_{\mathrm{HYP}}(r+a)+G_{\mathrm{HYP}}(r-a)-2 G_{\mathrm{HYP}}(r)\right] / a^{2} .
$$

In practice we evaluate the momentum integral in Eq. (A.2) by discrete momentum sums over $p_{j}=2 \pi n_{j} a / L, n_{j}=0,1, \ldots, L / a-1$. In Table 2 we quote the results for $r_{\mathrm{I}} / a$ and $\tilde{r} / a$ obtained from extrapolations $L / a \rightarrow \infty$. The latter are done with the method explained in Appendix D of [54] and we consider lattice sizes larger than $L / a=128$ up to $L / a=512$. Due to the symmetry under $p_{j} \rightarrow-p_{j}$ of the integrand only odd powers of $a / L$ can appear in the expansion in powers of $a / L$ and in general this evaluation of the integral is the application of a trapezoidal rule, which has discretization errors of order $(a / L)^{2}$. Thus the leading correction is $s_{1}(a / L)^{3}$. The data for $r_{\mathrm{I}} / a$ and $\tilde{r} / a$ are very well fitted by a polynomial $s_{0}+s_{1}(a / L)^{3}+s_{2}(a / L)^{5}$ and we added terms $s_{3}(a / L)^{7}+s_{4}(a / L)^{9}$ to estimate the systematic error of the extrapolations. In Table 2 we list the extrapolated values with six significant digits.

\section{B Perturbation theory for $c(r)$}

We consider QCD with $N_{\mathrm{f}}$ massless dynamical quark flavors. The quantity $c(r)$ in Eq. (1.2) defines a renormalized coupling $\left(C_{\mathrm{F}}=4 / 3\right)$,

$$
\bar{g}_{\mathrm{c}}^{2}(\mu)=-\frac{4 \pi}{C_{\mathrm{F}}} c(r), \mu=1 / r .
$$

It is very similar to $\bar{g}_{\mathrm{qq}}^{2}(\mu)=\frac{4 \pi}{C_{\mathrm{F}}} r^{2} F(r), \mu=1 / r$ discussed in [50]. The relation is

$$
\bar{g}_{\mathrm{c}}^{2}=\bar{g}_{\mathrm{qq}}^{2}+\bar{g}_{\mathrm{qq}} \beta_{\mathrm{qq}} \text {. }
$$

For a perturbative evaluation of one-scale quantities such as $c(r)$ it is natural 8 to just integrate the renormalization group equation

$$
\mu \frac{\mathrm{d}}{\mathrm{d} \mu} \bar{g}_{\mathrm{c}}(\mu)=\beta_{\mathrm{c}}\left(\bar{g}_{\mathrm{c}}(\mu)\right) \text {. }
$$

We do this in the precise form of

$$
\frac{\Lambda_{\mathrm{c}}}{\mu}=\left(b_{0} \bar{g}_{\mathrm{c}}^{2}\right)^{-b_{1} /\left(2 b_{0}^{2}\right)} \mathrm{e}^{-1 /\left(2 b_{0} \bar{g}_{\mathrm{c}}^{2}\right)} \exp \left\{-\int_{0}^{\bar{g}_{\mathrm{c}}} \mathrm{d} x\left[\frac{1}{\beta_{\mathrm{c}}(x)}+\frac{1}{b_{0} x^{3}}-\frac{b_{1}}{b_{0}^{2} x}\right]\right\},
$$

\footnotetext{
${ }^{8}$ It has also been observed in more than one case that it also yields a good perturbative description of the non-perturbative behavior.
} 
where for $\beta_{\mathrm{c}}$ the truncated perturbative expansion is inserted, but the integral is (numerically) evaluated as it stands. The Lambda-parameter in the c-scheme is

$$
\Lambda_{c}=\mathrm{e}^{-1 / 2} \Lambda_{\mathrm{qq}}=\Lambda_{\overline{\mathrm{MS}}} \mathrm{e}^{k_{1} /\left(8 \pi b_{0}\right)-1 / 2},
$$

where $k_{1}=\frac{1}{4 \pi}\left(a_{1}+a_{2} N_{\mathrm{f}}\right), a_{1}=-35 / 3+22 \gamma_{\mathrm{E}}$ and $a_{2}=2 / 9-4 \gamma_{\mathrm{E}} / 3$ [55]. We now turn to the perturbative beta function.

\section{B.1 Perturbative beta function in the c-scheme}

The expansion of the potential in the $\overline{\mathrm{MS}}$ coupling is now known to a high accuracy. After the $\bar{g} \frac{6}{\mathrm{MS}}$ term [56, 57], the resummation of the infrared divergent diagrams appearing first at the next order was performed [52,58, yielding a $\sim \bar{g}_{\overline{\mathrm{MS}}}^{8} \log \left(\bar{g}_{\overline{\mathrm{MS}}}^{2}\right)$ term. Recently also the full three-loop computation was finished by two groups [59,60]. Due to the $\bar{g}_{\overline{\mathrm{MS}}}^{8} \log \left(\bar{g}_{\overline{\mathrm{MS}}}^{2}\right)$ term in the potential [52,58, the beta function has a perturbative expansion

$$
\beta_{\mathrm{c}}\left(\bar{g}_{\mathrm{c}}\right)=-\bar{g}_{\mathrm{c}}^{3}\left[\sum_{n=0}^{3} b_{n}^{(\mathrm{c})} \bar{g}_{\mathrm{c}}^{2 n}+b_{3, l}^{(\mathrm{c})} \bar{g}_{\mathrm{c}}^{6} \log \left(C_{\mathrm{A}} \bar{g}_{\mathrm{c}}^{2} /(8 \pi)\right)+\mathrm{O}\left(\bar{g}_{\mathrm{c}}^{8}\right)\right],
$$

with the universal coefficients $\left(C_{\mathrm{A}}=3\right)$

$$
\begin{aligned}
& b_{0}^{(\mathrm{c})}=b_{0}=\frac{1}{(4 \pi)^{2}}\left(11 C_{\mathrm{A}} / 3-2 N_{\mathrm{f}} / 3\right), \\
& b_{1}^{(\mathrm{c})}=b_{1}=\frac{1}{(4 \pi)^{4}}\left(34 C_{\mathrm{A}}^{2} / 3-10 C_{\mathrm{A}} N_{\mathrm{f}} / 3-2 C_{\mathrm{F}} N_{\mathrm{f}}\right) .
\end{aligned}
$$

We now describe how the non-universal coefficients are obtained from the results in the literature. Our starting point is Eq. (40) of [61], which is the expansion of the static potential $V(r)$, denoted "static energy" in [61], in the $\overline{\mathrm{MS}}$ strong coupling $\alpha_{\mathrm{s}}=\bar{g}_{\overline{\mathrm{MS}}}^{2}(1 / r) /(4 \pi)$ derived from the above mentioned work. Introducing the notation $V(r)=-C_{\mathrm{F}} G\left(\alpha_{\mathrm{s}}\right) / r$, we obtain an expansion for $\alpha_{\mathrm{c}}=$ $\bar{g}_{\mathrm{c}}^{2} /(4 \pi)$ :

$$
\begin{aligned}
\alpha_{\mathrm{c}} & =\frac{1}{2} r^{2} G^{\prime \prime}\left(\alpha_{\mathrm{s}}\right)-r G^{\prime}\left(\alpha_{\mathrm{s}}\right)+G\left(\alpha_{\mathrm{s}}\right) \\
& =\alpha_{\mathrm{s}}+d_{1} \alpha_{\mathrm{s}}^{2}+d_{2} \alpha_{\mathrm{s}}^{3}+d_{3} \alpha_{\mathrm{s}}^{4}+d_{3, l} \alpha_{\mathrm{s}}^{4} \ln \left(\frac{C_{\mathrm{A}} \alpha_{\mathrm{s}}}{2}\right)+\mathcal{O}\left(\alpha_{\mathrm{s}}^{5}\right)
\end{aligned}
$$

where the primes in the first equation mean derivatives with respect to $r$ and the coefficients of the expansion are

$$
d_{1}=\frac{1}{4 \pi}\left(\tilde{a}_{1}-3 b_{0}(4 \pi)^{2}\right)
$$




$$
\begin{aligned}
d_{2}= & \frac{1}{(4 \pi)^{2}}\left(\tilde{a}_{2, s}+4 b_{0}^{2}(4 \pi)^{4}-3 b_{1}(4 \pi)^{4}-6 \tilde{a}_{1} b_{0}(4 \pi)^{2}\right) \\
d_{3}= & \frac{1}{(4 \pi)^{3}}\left(a_{3}+12 \tilde{a}_{1} b_{0}^{2}(4 \pi)^{4}-6 \tilde{a}_{1} b_{1}(4 \pi)^{4}+10 b_{0} b_{1}(4 \pi)^{6}\right. \\
& \left.-3 b_{2}(4 \pi)^{6}-9 \tilde{a}_{2, s} b_{0}(4 \pi)^{2}\right) \\
d_{3, l}= & \frac{C_{\mathrm{A}}^{3}}{12 \pi}
\end{aligned}
$$

The coefficients $b_{2}$ and $b_{3}$ of the beta function in the $\overline{\mathrm{MS}}$ scheme, $\beta_{\overline{\mathrm{MS}}}\left(\bar{g}_{\overline{\mathrm{MS}}}\right) \sim$ $-\bar{g}_{\overline{\mathrm{MS}}}^{3} \sum_{n \geq 0} \bar{g}_{\overline{\mathrm{MS}}}^{2 n} b_{n}$, can be found in [62,63]. The coefficient $\tilde{a}_{1}$ is defined in Eq. (7) and the coefficient $\tilde{a}_{2, s}$ in Eq. (8) of [61], they both depend on $N_{\mathrm{f}}$. The coefficient $a_{3}$ is

$$
\begin{aligned}
a_{3}= & \frac{4^{4}}{3 C_{\mathrm{F}}}\left(c_{0}\left(N_{\mathrm{f}}\right)+2 \gamma_{\mathrm{E}} c_{1}\left(N_{\mathrm{f}}\right)+\left(4 \gamma_{\mathrm{E}}^{2}+\pi^{2} / 3\right) c_{2}\left(N_{\mathrm{f}}\right)\right. \\
& \left.+\left(8 \gamma_{\mathrm{E}}^{3}+2 \pi^{2} \gamma_{\mathrm{E}}+16 \zeta(3)\right) c_{3}\left(N_{\mathrm{f}}\right)\right)
\end{aligned}
$$

where

$$
c_{0}\left(N_{\mathrm{f}}\right)=219.59+\left(a_{3}^{(1)} N_{\mathrm{f}}+a_{3}^{(2)} N_{\mathrm{f}}^{2}+a_{3}^{(3)} N_{\mathrm{f}}^{3}\right) / 4^{3} .
$$

From [61,64] we get9 $c_{0}(0)$ and the coefficients $a_{3}^{(1)}, a_{3}^{(2)}$ and $a_{3}^{(3)}$ are given in Eq. (6) of [65]. The coefficients $c_{1}\left(N_{\mathrm{f}}\right), c_{2}\left(N_{\mathrm{f}}\right)$ and $c_{3}\left(N_{\mathrm{f}}\right)$ are defined in Eqs. (10), (11) and (12) of [66] respectively.

The non-universal coefficients $b_{2}^{(\mathrm{c})}$ and $b_{3}^{(\mathrm{c})}$ as well as the coefficient $b_{3, l}^{(\mathrm{c})}$ may now be computed by differentiating $\beta_{\mathrm{c}}=\frac{2 \pi}{\bar{g}_{\mathrm{c}}} \mu \frac{\mathrm{d} \alpha_{\mathrm{c}}}{\mathrm{d} \mu}$ with $\alpha_{\mathrm{c}}$ of Eq. (B.10), where the $\overline{\mathrm{MS}}$ beta function is used. This first yields $\beta_{\mathrm{c}}$ as a function of $\alpha_{\mathrm{s}}$ from which we change to $\beta_{\mathrm{c}}\left(\bar{g}_{\mathrm{c}}\right)$ by inserting the inverted Eq. (B.10), $\alpha_{\mathrm{s}}=\alpha_{\mathrm{c}}+\ldots-$ $d_{3, l} \alpha_{\mathrm{c}}^{4} \ln \left(\frac{C_{\mathrm{A}} \alpha_{\mathrm{c}}}{2}\right)$.

Carrying this out in MAPLE we find

$$
\begin{aligned}
b_{2}^{(\mathrm{c})}= & b_{2}-5 b_{0}^{3}+\tilde{a}_{2, s} b_{0}(4 \pi)^{-4}-\tilde{a}_{1} b_{1}(4 \pi)^{-2}-\tilde{a}_{1}^{2} b_{0}(4 \pi)^{-4} \\
= & (4 \pi)^{-3}\left[0.98165-0.16738 N_{\mathrm{f}}-0.00212 N_{\mathrm{f}}^{2}+0.00026 N_{\mathrm{f}}^{3}\right] \\
b_{3}^{(\mathrm{c})}= & b_{3}-2 \tilde{a}_{1} b_{2}(4 \pi)^{-2}+2 a_{3} b_{0}(4 \pi)^{-6}+\frac{1}{3} C_{\mathrm{A}}^{3} b_{0}(4 \pi)^{-4}-25 b_{0}^{2} b_{1} \\
& -6 \tilde{a}_{1} \tilde{a}_{2, s} b_{0}(4 \pi)^{-6}+\tilde{a}_{1}^{2} b_{1}(4 \pi)^{-4}-36 b_{0}^{4}+4 \tilde{a}_{1}^{3} b_{0}(4 \pi)^{-6} \\
= & (4 \pi)^{-4}\left[0.12206+0.09696 N_{\mathrm{f}}-0.01899 N_{\mathrm{f}}^{2}+0.0004458 N_{\mathrm{f}}^{3}\right. \\
& \left.+0.0000195 N_{\mathrm{f}}^{4}\right] \\
b_{3, l}^{(\mathrm{c})}= & \frac{2}{3} C_{\mathrm{A}}^{3} b_{0}(4 \pi)^{-4}=(4 \pi)^{-4}\left[1.25385-0.07599 N_{\mathrm{f}}\right] .
\end{aligned}
$$

\footnotetext{
${ }^{9}$ We thank the authors of [61] for communication on the value of $c_{0}(0)$.
} 


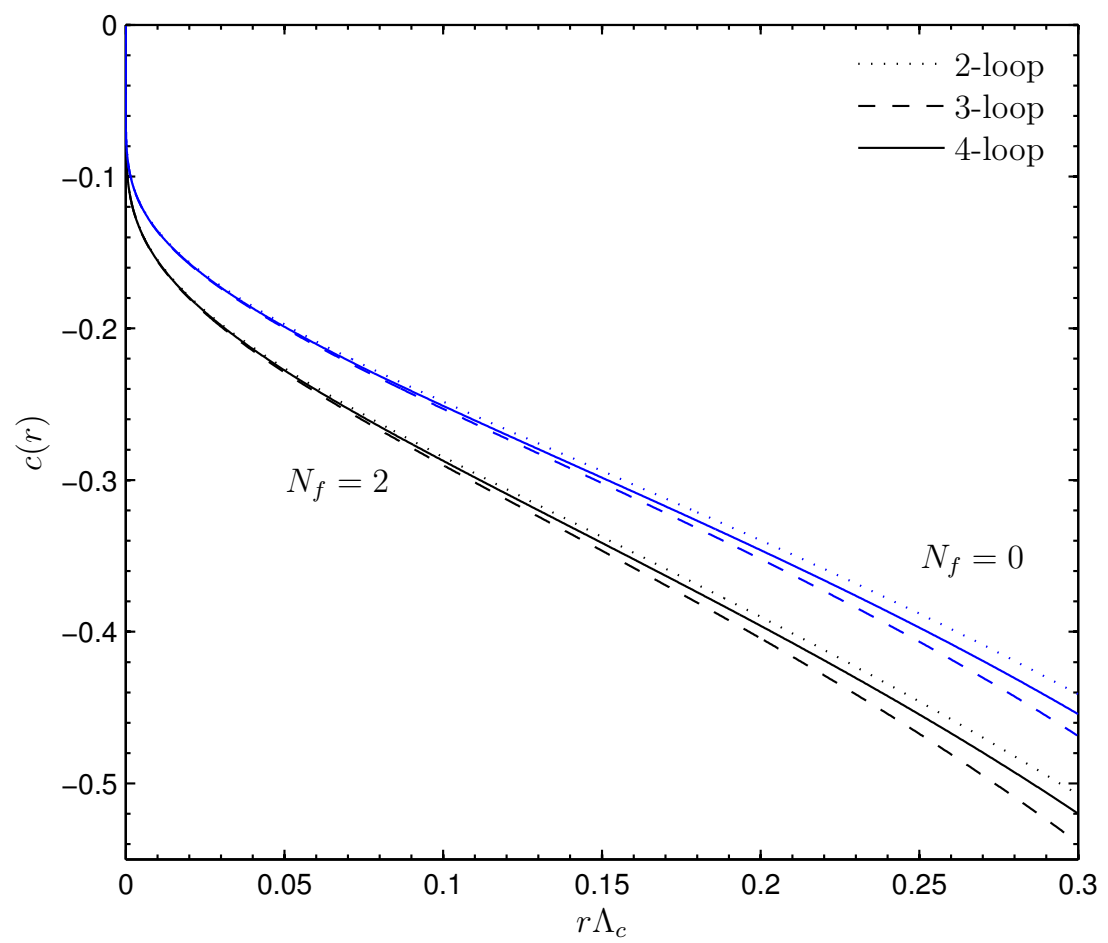

Figure 8: The perturbative running of the quantity $c(r)$ obtained from Eq. (B.4) using the 2-loop (dotted lines), 3-loop (dashed lines) and 4-loop (continued lines) beta function $\beta_{\mathrm{c}}\left(\bar{g}_{\mathrm{c}}\right)$ for $N_{\mathrm{f}}=0,2$.

As in the $\overline{\mathrm{MS}}$ scheme the coefficients $(4 \pi)^{n+1} b_{n}$ are of order one and thus the beta fuction has a well-behaved expansion up to couplings $\alpha_{\mathrm{c}}$ of the order of $1 / 3$. The perturbative running of $c(r)$ is shown in Fig. 8.

The "asymptotic convergence" of the series Eq. (B.10) is not good. It can be substantially improved by matching the couplings at a different scale, i.e., by expressing $\alpha_{\mathrm{c}}(s / r)$ as a function of $\alpha_{\mathrm{s}}(1 / r)$ and choosing $s=s_{0}=\Lambda_{c} / \Lambda_{\overline{\mathrm{MS}}}$ ("fastest apparent convergence", cf. [50, 55]). The resulting curves for $\alpha_{\mathrm{c}}$ are hardly distinguishable from the ones shown in Fig. 8.

\section{B.2 Perturbative beta function in the qq-scheme}

In the same way one obtains the beta function in the qq-scheme. We update the formulae given in [55] to include the 4-loop term:

$$
\begin{aligned}
b_{2}^{(\mathrm{qq})}= & (4 \pi)^{-3}\left[1.6524-0.28933 N_{\mathrm{f}}+0.00527 N_{\mathrm{f}}^{2}+0.00011 N_{\mathrm{f}}^{3}\right] \\
b_{3}^{(\mathrm{qq})}= & (4 \pi)^{-4}\left[4.94522-1.07965 N_{\mathrm{f}}+0.079107 N_{\mathrm{f}}^{2}-0.002774 N_{\mathrm{f}}^{3}\right. \\
& \left.+0.000051 N_{\mathrm{f}}^{4}\right]
\end{aligned}
$$




$$
b_{3, l}^{(\mathrm{qq})}=\frac{2}{3} C_{\mathrm{A}}^{3} b_{0}(4 \pi)^{-4}=(4 \pi)^{-4}\left[1.25385-0.07599 N_{\mathrm{f}}\right] .
$$

Perturbation theory in the c-scheme appears much better behaved than in the qq-scheme. Since this can only be considered an accident we come to the same conclusion as [55], namely that the perturbative description of the static potential is accurately valid only at rather small values of $r$, where $\alpha_{\mathrm{qq}}(1 / r) \approx 1 / 4$. Unfortunately these distances are close to present lattice spacings. In particular the data presented in this paper are not good enough to extract the $\Lambda$ parameter through Eq. (B.4) or variants thereof.

\section{References}

[1] G. Parisi, R. Petronzio and F. Rapuano, Phys. Lett. B128 (1983) 418.

[2] M. Lüscher and P. Weisz, JHEP 09 (2001) 010, hep-lat/0108014.

[3] A. Hasenfratz and F. Knechtli, Phys.Rev. D64 (2001) 034504, heplat/0103029.

[4] A. Hasenfratz, R. Hoffmann and F. Knechtli, Nucl.Phys.Proc.Suppl. 106 (2002) 418, hep-lat/0110168.

[5] C. Alexandrou, P. De Forcrand and A. Tsapalis, Phys. Rev. D65 (2002) 054503, hep-lat/0107006.

[6] ALPHA Collaboration, M. Della Morte et al., Phys.Lett. B581 (2004) 93, hep-lat/0307021.

[7] M. Della Morte, A. Shindler and R. Sommer, JHEP 0508 (2005) 051, heplat/0506008.

[8] A. Grimbach, D. Guazzini, F. Knechtli and F. Palombi, JHEP 0803 (2008) 039, arXiv:0802.0862.

[9] R. Sommer, Nucl.Phys. B411 (1994) 839, hep-lat/9310022.

[10] J.M. Maldacena, Phys. Rev. Lett. 80 (1998) 4859, hep-th/9803002.

[11] V. Forini, JHEP 11 (2010) 079, arXiv:1009.3939.

[12] J.K. Erickson, G.W. Semenoff and K. Zarembo, Nucl. Phys. B582 (2000) 155, hep-th/0003055. 
[13] A. Pineda, Phys. Rev. D77 (2008) 021701, arXiv:0709.2876.

[14] K. Schilling, Nucl. Phys. Proc. Suppl. 83 (2000) 140, hep-lat/9909152.

[15] SESAM, G.S. Bali, H. Neff, T. Duessel, T. Lippert and K. Schilling, Phys. Rev. D71 (2005) 114513, hep-lat/0505012.

[16] M. Pepe, (2010), arXiv:1011.0056.

[17] R. Sommer, Phys. Rept. 275 (1996) 1, hep-lat/9401037.

[18] M. Lüscher and P. Weisz, JHEP 0207 (2002) 049, hep-lat/0207003.

[19] M. Lüscher, Commun. Math. Phys. 54 (1977) 283.

[20] E. Eichten and B.R. Hill, Phys. Lett. B234 (1990) 511.

[21] R. Sommer, (2010), arXiv:1008.0710.

[22] F. Knechtli, (1999), hep-lat/9910044.

[23] S. Necco and R. Sommer, Nucl. Phys. B622 (2002) 328, hep-lat/0108008.

[24] ALPHA, M. Kurth and R. Sommer, Nucl. Phys. B597 (2001) 488, heplat/0007002.

[25] B. Sheikholeslami and R. Wohlert, Nucl. Phys. B259 (1985) 572.

[26] M. Lüscher, S. Sint, R. Sommer and P. Weisz, Nucl. Phys. B478 (1996) 365, hep-lat/9605038.

[27] ALPHA, K. Jansen and R. Sommer, Nucl. Phys. B530 (1998) 185, heplat/9803017.

[28] ALPHA, R. Frezzotti, P.A. Grassi, S. Sint and P. Weisz, JHEP 08 (2001) 058, hep-lat/0101001.

[29] R. Frezzotti and G.C. Rossi, JHEP 08 (2004) 007, hep-lat/0306014.

[30] B. Blossier et al., PoS (Lattice 2010) 308 (2010), arXiv:1012.1357.

[31] ALPHA collaboration, M. Guagnelli, R. Sommer and H. Wittig, Nucl.Phys. B535 (1998) 389, hep-lat/9806005.

[32] C.W. Bernard and T.A. DeGrand, Nucl. Phys. Proc. Suppl. 83 (2000) 845, hep-lat/9909083. 
[33] N.A. Campbell, A. Huntley and C. Michael, Nucl. Phys. B306 (1988) 51.

[34] M. Lüscher and U. Wolff, Nucl. Phys. B339 (1990) 222.

[35] B. Blossier, M. Della Morte, G. von Hippel, T. Mendes and R. Sommer, JHEP 04 (2009) 094, arXiv:0902.1265.

[36] O. Philipsen and H. Wittig, Phys. Rev. Lett. 81 (1998) 4056, Erratumibid.83:2684,1999, hep-lat/9807020.

[37] ALPHA, F. Knechtli and R. Sommer, Phys. Lett. B440 (1998) 345, Erratumibid.B454:399,1999, hep-lat/9807022.

[38] ALPHA, F. Knechtli and R. Sommer, Nucl. Phys. B590 (2000) 309, heplat/0005021.

[39] F. Gliozzi and A. Rago, Nucl.Phys. B714 (2005) 91, hep-lat/0411004.

[40] P. Weisz, Nucl.Phys. B212 (1983) 1.

[41] M. Lüscher, Comput. Phys. Commun. 165 (2005) 199, hep-lat/0409106.

[42] M. Lüscher, JHEP 12 (2007) 011, 0710.5417.

[43] B. Leder and F. Knechtli, (2010), arXiv:1012.1141.

[44] S. Schaefer, R. Sommer and F. Virotta, (2010), arXiv:1009.5228.

[45] ALPHA, U. Wolff, Comput. Phys. Commun. 156 (2004) 143, Erratumibid.176:383,2007, hep-lat/0306017.

[46] E. Eichten, K. Gottfried, T. Kinoshita, K.D. Lane and T.M. Yan, Phys. Rev. D21 (1980) 203.

[47] J.L. Richardson, Phys. Lett. B82 (1979) 272.

[48] M. Lüscher, K. Symanzik and P. Weisz, Nucl. Phys. B173 (1980) 365.

[49] M. Lüscher, Nucl. Phys. B180 (1981) 317.

[50] S. Necco and R. Sommer, Phys.Lett. B523 (2001) 135, hep-ph/0109093.

[51] Quarkonium Working Group, N. Brambilla et al., (2004), hep-ph/0412158, Published as CERN Yellow Report, CERN-2005-005, Geneva: CERN, 2005, $487 \mathrm{p}$. 
[52] N. Brambilla, A. Pineda, J. Soto and A. Vairo, Nucl.Phys. B566 (2000) 275, hep-ph/9907240.

[53] R. Hoffmann, Diploma Thesis (2002).

[54] ALPHA, A. Bode, P. Weisz and U. Wolff, Nucl. Phys. B576 (2000) 517, hep-lat/9911018.

[55] S. Necco, (2003), hep-lat/0306005, Ph.D. Thesis.

[56] M. Peter, Nucl. Phys. B501 (1997) 471, hep-ph/9702245.

[57] Y. Schröder, Phys. Lett. B447 (1999) 321, hep-ph/9812205.

[58] N. Brambilla, A. Pineda, J. Soto and A. Vairo, Phys.Rev. D60 (1999) 091502, hep-ph/9903355.

[59] A.V. Smirnov, V.A. Smirnov and M. Steinhauser, Phys.Rev.Lett. 104 (2010) 112002, arXiv:0911.4742.

[60] C. Anzai, Y. Kiyo and Y. Sumino, Phys.Rev.Lett. 104 (2010) 112003, arXiv:0911.4335.

[61] N. Brambilla, A. Vairo, X. Garcia i Tormo and J. Soto, Phys.Rev. D80 (2009) 034016, arXiv:0906.1390.

[62] T. van Ritbergen, J. Vermaseren and S. Larin, Phys.Lett. B400 (1997) 379, hep-ph/9701390.

[63] M. Czakon, Nucl.Phys. B710 (2005) 485, hep-ph/0411261.

[64] N. Brambilla, X. Garcia i Tormo, J. Soto and A. Vairo, Phys.Rev.Lett. 105 (2010) 212001, arXiv:1006.2066.

[65] A.V. Smirnov, V.A. Smirnov and M. Steinhauser, Phys.Lett. B668 (2008) 293, arXiv:0809.1927.

[66] F. Chishtie and V. Elias, Phys.Lett. B521 (2001) 434, hep-ph/0107052. 